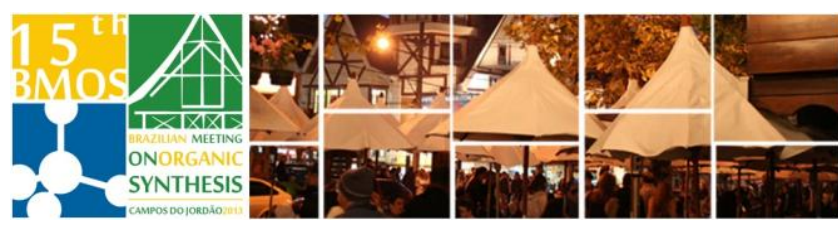

\title{
A versatile synthesis to trisubstituted nitroalkenes via nitroaldol reaction: Synthesis of Baylis-Hillman type adducts
}

\author{
Vera L. Patrocinio Pereira,${ }^{1 *}$ Jeronimo da Silva Costa,${ }^{2}$ Alessandra C. de Souza e \\ Silva, ${ }^{2}$ Fernanda Cunha do Nascimento. ${ }^{2}$
}

${ }^{1}$ Universidade Federal do Rio de Janeiro, Núcleo de Pesquisas de Produtos Naturais, 21941-902, Rio de Janeiro, RJ, Brazil. ' Instituto Federal de Educação, Ciência e Tecnologia do Rio de Janeiro, 26530-060, Nilópolis, RJ, Brazil. *patrocinio@nppn.ufrj.br

Keywords: Henry reaction, Baylis-Hillman adducts, Nitroalkanes.

\section{INTRODUCTION}

Electron deficient nitroalkenes consists of a versatile class of compounds reactives in Michael, FriedelCrafts, Baylis-Hillman, Diels-Alder, Hetero-DielsAlder, [3+2]-cycloaddition and Cross Coupling reactions. ${ }^{1}$ Usually, $\beta$-monosubstituted nitroalkenes and $\alpha, \beta$-disubstituted nitroalkenes are synthesized via a nitroaldol reaction between an aldehyde and the nitromethane or a primary nitroalkane, respectively. The hydroxyl group elimination, in its free or active form, leads to the desired nitroalkene. Another usual route consists of nitration of the corresponding nitroalkene with toxic or volatile reagents leading to low regioselectivity. A more convenient method $\mathrm{NaNO}_{2}$-ceric ammonium nitrate has been reported. ${ }^{2}$ In the specific case of trisubstituted nitroalkenes, the nitroaldol route is not effective because of the high reversibility of nitroaldol reaction when ketones are employed.

\section{RESULTS AND DISCUSSION}

Here in, we report our preliminary results towards synthesis of various trisubstituted nitroalkenes 1 (Baylis-Hillman adducts) from the nitroaldol reaction betweew the acetylated nitroalcohols 4a-f and various aldehydes 2 followed by $\mathrm{AcOH}$ elimination Scheme 1. The acetylated nitroalcohols $4 a-f$ could be obtained via a nitroaldol reaction between diverse ketones (propanone (3a), cyclopentanone (3b), cyclohexanone (3c), 2-pentanone (3d), 2-butanone (3e) and acetophenone (3f)) base catalyzed and the nitromethane, followed by acetylation acid catalyzed.

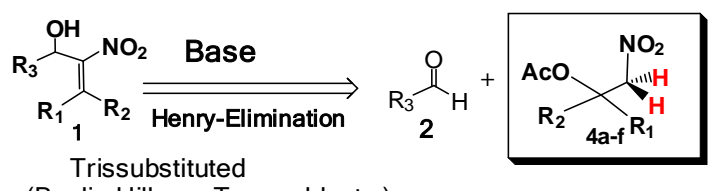

(Baylis-Hillman Type adducts ) Acetylated nitroalcohols

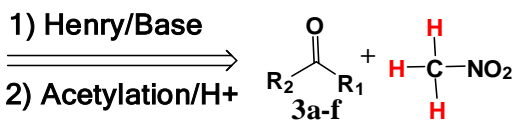

Scheme 1: Retrosynthesis to obtainment of 1.

The results summarized in the table 1 shows that the nitroesters derived from propanone, cyclohexanone and 2-pentanone were obtained in good yields (entries 1,3,4). Cyclopentanone (3b) and 2-butanone (3e) furnished $4 \mathrm{~b}$ and $4 \mathrm{e}$ in low yield (Entries 2,5). Acetophenone (3f) was not reactive (Entry 6).

Table 1:. Synthesis of acetylated nitroalcohols 4a-f.

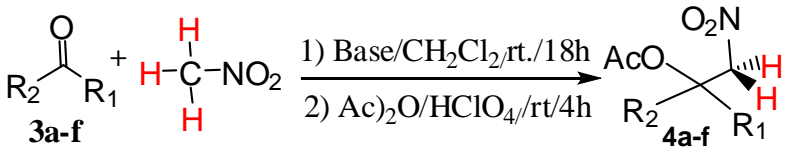

\begin{tabular}{|c|c|c|c|}
\hline Entry & \multicolumn{1}{|c|}{ Ketone } & Base & $\begin{array}{c}\text { Nitroester } \\
\text { Yield }(\%)^{\mathrm{a}}\end{array}$ \\
\hline 1 & 3a, $\mathrm{R}_{1}=\mathrm{R}_{2}=\mathrm{Me}$ & TBAF $^{\mathrm{D}}$ & $\mathbf{4 a / 5 0}$ \\
3 & $\mathbf{3 b}, \mathrm{R}_{1}=\mathrm{R}_{2}=\left(\mathrm{CH}_{2}\right)_{4}$ & $\mathrm{DBU}^{\mathrm{C}}$ & $\mathbf{4 b / 2 1}$ \\
4 & $\mathbf{3 c}, \mathrm{R}_{1}=\mathrm{R}_{2}=\left(\mathrm{CH}_{2}\right)_{5}$ & TBAF & $\mathbf{4 c / 6 5}$ \\
5 & $\mathbf{3 d}, \mathrm{R}_{1}=\mathrm{Me}, \mathrm{R}_{2}=\mathrm{Pr}$ & DBU & $\mathbf{4 d} / \mathbf{7 0}$ \\
6 & $\mathbf{3 e}, \mathrm{R}_{1}=\mathrm{Me}, \mathrm{R}_{2}=\mathrm{Et}$ & TBAF & $\mathbf{4 e / 3 0}$ \\
& & TBAF & No reaction \\
\hline
\end{tabular}

a) After column chromatographic (2 steps). b) 0.5 eq c) 1 eq. Reaction of $4 \mathbf{a}$ with (R)-glyceraldehyde acetonide (7) and 4d with butanal, via a nitroaldol-elimination sequence, produced the trisubstituted nitroalkenes 5 and 6 (Baylis-Hillman-type adducts) in $80 \%$ yield.

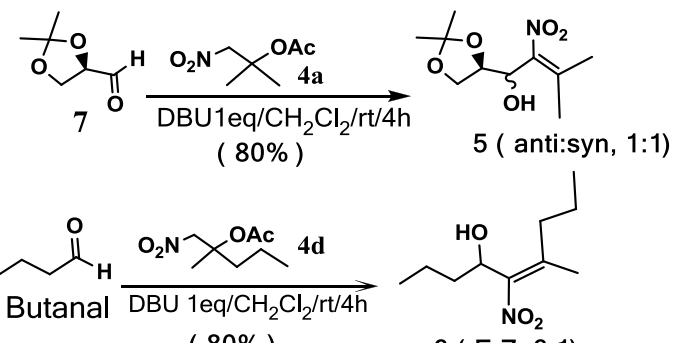

$$
(80 \%) \quad 6(\mathrm{E}: \mathrm{Z}, 6: 1)
$$

\section{CONCLUSION}

A versatile, simple and efficient route to produce diverse trisubstituted nitroalkenes has been attained.

\section{REFERENCES}

${ }^{1}$ Averina, B. E. et al. Tetrahedron. Lett. 2011, 52, 2910-2913.

${ }^{2}$ Pereira, V.L.P. et al. Beilstein J. Org. Chem 2013, 9, 832-837.

${ }^{3}$ Pereira,V.L.P. et al. Beilstein J. Org. Chem 2013, 9, 838-845. 